\title{
A new UHPLC-MS/MS method for the screening of urinary oligosaccharides expands the detection of storage disorders
}

\author{
Michela Semeraro ${ }^{1 *}+\left(\mathbb{D}\right.$, Elisa Sacchetti ${ }^{1 \dagger}$, Federica Deodato ${ }^{1}$, Turgay Coşkun ${ }^{2}$, Incilay Lay ${ }^{3}$, Giulio Catesini ${ }^{1}$, \\ Giorgia Olivieri ${ }^{1}$, Cristiano Rizzo ${ }^{1}$, Sara Boenzi ${ }^{1}$ and Carlo Dionisi-Vici ${ }^{1}$
}

\begin{abstract}
Background: Oligosaccharidoses are storage disorders due to enzymatic defects involved in the breakdown of the oligosaccharidic component of glycosylated proteins. The defect cause the accumulation of oligosaccharides (OS) and, depending on the lacking enzyme, results in characteristic profiles which are helpful for the diagnosis. We developed a new tandem mass spectrometry method for the screening of urinary OS which was applied to identify a large panel of storage disorders.
\end{abstract}

Methods: The method was set-up in urine and dried urine spots (DUS). Samples were analysed, without derivatization and using maltoheptaose as internal standard, by UHPLC-MS/MS with MRM acquisition of target OS transitions, including Glc4, the biomarker of Pompe disease. The chromatographic run was $<30 \mathrm{~min}$. Samples from patients with known storage disorders were used for clinical validation.

Results: The method allowed to confirm the diagnosis of oligosaccharidoses (sialidosis, a-/ $\beta$-mannosidosis, fucosidosis, aspartylglucosaminuria) and of GM1 and GM2 (Sandhoff type) gangliosidosis, by detecting specific OS profiles. In other storage disorders (mucolipidosis II and III, mucopolysaccharidosis type IVB) the analyisis revealed abnormal OS excretion with non-specific profiles. Besides Pompe disease, the tetrasaccharide Glc4 was increased also in disorders of autophagy (Vici syndrome, Yunis-Varon syndrome, and Danon disease) presenting cardiomuscular involvement with glycogen storage. Overall, results showed a clear separation between patients and controls, both in urine and in DUS.

Conclusion: This new UHPLC/MS-MS method, which is suitable for rapid and easy screening of OS in urine and DUS, expands the detection of storage disorders from oligosaccharidoses to other diseases, including the novel category of inherited disorders of autophagy.

Keywords: Oligosaccharides, Storage disorders, Pompe disease, Autophagy, Danon disease, Vici syndrome, Yunisvaron syndrome

\footnotetext{
*Correspondence: michela.semeraro@opbg.net

${ }^{\dagger}$ Michela Semeraro and Elisa Sacchetti equally contributed to this work

${ }^{1}$ Division of Metabolism and Metabolic Diseases Research Unit, Bambino

Gesù Children's Hospital, IRCCS, Viale San Paolo 15, 00146 Rome, Italy

Full list of author information is available at the end of the article
}

\section{Background}

The new classification of storage disorders includes nine disease categories (i.e. oligosaccharidoses, mucolipidoses, mucopolysaccharidoses, sphingolipidoses, neuronal ceroid lipofuscinosis, disorders of lysosomal cholesterol metabolism, disorders of lysosomal transport or sorting, disorders of lysosomal protein degradation, and the recently identified inherited disorders of autophagy) and original author(s) and the source, provide a link to the Creative Commons licence, and indicate if changes were made. The images or other third party material in this article are included in the article's Creative Commons licence, unless indicated otherwise in a credit line to the material. If material is not included in the article's Creative Commons licence and your intended use is not permitted by statutory regulation or exceeds the permitted use, you will need to obtain permission directly from the copyright holder. To view a copy of this licence, visit http://creativecommons.org/licenses/by/4.0/. The Creative Commons Public Domain Dedication waiver (http://creativeco mmons.org/publicdomain/zero/1.0/) applies to the data made available in this article, unless otherwise stated in a credit line to the data. 
accounts for over 65 different inherited disorders [1]. Oligosaccharidoses are due to defects of lysosomal enzymes involved in the catabolic pathway for the breakdown of the oligosaccharidic component of glycosylated proteins [2]. The glycosidic groups, composed of fucose, mannose, sialic acid, galactose, and $\mathrm{N}$-acetylglucosamine residues, to form glycoproteins are either N-linked (to asparagine) or O-linked (to serine or threonine) [3]. Enzymatic defects of this catabolic pathway cause the accumulation of oligosaccharides (OS) and, depending on the lacking enzyme, result in characteristic profiles of urinary OS, which are helpful for the diagnosis [4]. The different oligosaccharidoses share common clinical features, which include facial dymorphisms, dysostosis multiplex, hepato/splenomegaly, developmental delay and neurological signs, making difficult the differential diagnosis [2]. Biochemically, OS analysis is the first step for the diagnosis of oligosaccharidoses [5]. The initial methods to analyze urinary OS were based on thin layer chromatography (TLC), but this assay has limited analytical sensitivity and specificity due to interfering compounds derived, especially in early infancy, from the diet or from medications [5-7].

A more powerful tool for the analysis of OS has been provided thanks to the introduction in diagnostic laboratories of mass spectrometry (MS), which allows to characterize the different OS species through specific multiple reaction monitoring (MRM) transitions. The analysis with a triple quadrupole after sample derivatization enabled the detection of characteristic OS profiles in the urine of patients with different types of oligosaccharidoses and with other storage disorders, and was also suitable for prenatal diagnosis in amniotic fluid [3, 8, 9]. Other methods, utilizing capillary high performance anion-exchange chromatography mass spectrometry (HPAEC) or matrix-assisted laser desorption ionization time-of-flight (MALDI-TOF/TOF), with or without sample derivatization, have been developed for structural studies and for disease screening [10, 12]. Piraud et al. adapted a MALDI-TOF/TOF based method [12] to a triple quadrupole analyzer, and built a powerful technique suitable in diagnostic laboratory for the screening in urine of a large number of oligosaccharidoses and for prenatal diagnosis in amniotic fluid [4]. More recently, an ultra-high performance liquid chromatography mass spectrometry (UHPLC-MS/MS) method for urinary OS analysis requiring sample derivatization has been reported [13]. The rapid evolution of all these techniques has potentially expanded the list of identifiable diseases to other storage disorders which share with oligosaccharidoses an abnormal excretion of compounds related to this catabolic pathway [3, 4, 8-13]. Furthermore, in Pompe disease, a glycogen storage disorder due to deficiency of the lysosomal enzyme acid $\alpha$-glucosidase, the profile of urinary OS by TLC analysis shows the presence of a large band which was characterized by UHPLC-MS/MS analysis as the tetrasaccharide 6- $\alpha$-Dglucopyranosyl-maltotriose (Glc $\alpha$ 1-6Glc $\alpha$ 1-4Glc $\alpha$ 1-4Glc, designed as Glc4) [14, 15]. More recently, Glc4 has been suggested as a target biomarker for diagnosis, monitoring disease progression and to evaluate the response to enzyme replacement therapy in Pompe disease $[16,17]$. Indeed, an abnormal OS profile by TLC analysis $[18,19]$ as been reported in Yunis-Varon syndrome, an inherited disorder of autophagy, a novel disease group listed among storage disorders [1].

In this study, we report a new UHPLC-MS/MS method, not requiring sample derivatization, for the screening of OS in urine and in dried urine spots (DUS) which was applied to identify a large panel of storage disorders.

\section{Methods}

Samples collection and complience with ethic guidelines Controls' and patients' urines and DUS samples were collected after obtaining informed consent. The work has been carried out in accordance with "The Code of Ethics of the World Medical Association (Declaration of Helsinki) for experiments involving humans"; "Uniform Requirements for manuscripts submitted to Biomedical journals" published by the International Committee of Medical Journal Editors. Samples were obtained from patients-followed by the Division of Metabolism, Bambino Gesù Childrens Hospital in Rome, Italy and by the Department of Pediatrics, Metabolism Unit, Hacettepe University, Ankara, Turkey-with a confirmed diagnosis made through enzymatic and/or genetic analysis or from positive quality controls provided by the European Research Network for evaluation and improvement of screening, Diagnosis and treatment of Inherited disorders of Metabolism (ERNDIM). Urine samples $(n=42)$ were obtained from 27 patients, age 7 months- 17 years, affected by the following storage disorders: sialidosis $(n=1), \alpha$-mannosidosis $(n=2)$, $\beta$-mannosidosis $(n=1)$, fucosidosis $(n=3)$, aspartylglucosaminuria $(n=1)$, GM1 gangliosidosis $(n=14)$, GM2 gangliosidosis $(n=6)$, mucolipidosis type II $(n=3)$ and mucolipidosis type III $(n=2)$, Pompe disease $(n=4)$, Vici syndrome $(n=4)$, Danon disease $(n=1)$. Samples were kept frozen at $-20{ }^{\circ} \mathrm{C}$ until analysis. DUS samples $(n=29)$ were obtained from 25 patients, age $1-18$ years, affected by the following storage disorders: sialidosis $(\mathrm{n}=1), \alpha$-mannosidosis $(\mathrm{n}=3), \beta$-mannosidosis $(\mathrm{n}=1)$, fucosidosis $(\mathrm{n}=3)$, aspartylglucosaminuria $(\mathrm{n}=1)$, GM1 gangliosidosis $(\mathrm{n}=4)$, GM2 gangliosidosis $(\mathrm{n}=1)$, mucolipidosis type II $(n=1)$, mucolipidosis type III $(n=2)$, mucopolysaccharidosis type IVb $(n=5)$, Pompe 
disease $(\mathrm{n}=2)$, Vici syndrome $(\mathrm{n}=3)$ and Yunis-Varon syndrome $(n=1)$, Danon disease $(n=1)$. The control urine $(n=75)$, age 2 months- 17 years and DUS $(n=12)$, age 1 months -18 years, samples were obtained from healthy subjects referred for routine urine laboratory analysis. Urine samples were kept at $-20{ }^{\circ} \mathrm{C}$ and DUS at room temperature until analysis.

A positive internal quality control (iQC) mix, containing an equal part of lyophilized urine from patients affected by sialidosis, $\alpha$-mannosidosis, $\beta$-mannosidosis, fucosidosis, aspartylglucosaminuria, GM1 gangliosidosis, GM2 gangliosidosis, Pompe disease provided by ERNDIM, was reconstituted, aliquoted and stored at $-20^{\circ} \mathrm{C}$.

\section{Urine samples treatment}

Urine samples were ultra-filtered with an Amicon Ultra filter $0.5 \mathrm{~mL} 3 \mathrm{~K} 96$ PK (Merck KGaA, Darmstadt, Germany) and centrifuged for $9 \mathrm{~min}$ at $13.000 \mathrm{rpm}$. Ultrafiltered urine were diluted with milli-Q water (Milli-Q Advantage A10 System, Merckmillipore, Merck KGaA, Darmstadt, Germany) to obtain a final creatinine concentration of $1 \mathrm{mM}$. The creatinine concentration was determined with the Jaffé method. Samples with a creatinine concentration $<1 \mathrm{mmol} / \mathrm{L}$ were not diluted and were adjusted at a creatinine concentration of $1 \mathrm{mM}$ after the analysis.

Fifty $\mu \mathrm{L}$ of normalized ultra-filtered urine were mixed in a glass tube with $20 \mu \mathrm{L}$ of the internal standard (IS) working solution [maltoheptaose (Glc7) $170 \mu \mathrm{mol} / \mathrm{L}$ dissolved in $\mathrm{H}_{2} \mathrm{O}$ ], $130 \mu \mathrm{L}$ of reconstitution buffer [37.5\% acetonitrile $(\mathrm{ACN}) / \mathrm{H}_{2} \mathrm{O}$ containing $0.02 \%$ formic acid $(\mathrm{FOA})(\mathrm{v} / \mathrm{v})]$, and vortexed.

\section{Dried urine spot (DUS) samples treatment}

To prepare DUS samples $2 \mathrm{ml}$ of urine were spotted, both in the laboratory and in the clinic, on the entire absorbing part of the card commonly used for newborn screening (EBF Eastern Business Forms, INC., SC, US) and dried at room temperature for at least $2 \mathrm{~h}$. DUS were shredded and mixed in deionized water for $30 \mathrm{~min}$ for the extraction. The entire absorbing part of the newborn screening card was used for the extraction. The extract was then diluted with Milli-Q water to obtain a creatinine concentration of $1 \mathrm{mM}$. The extract creatinine concentration was determined with the Jaffé method. Extracts with a creatinine concentration $<1 \mathrm{mmol} / \mathrm{L}$ were not diluted and were adjusted at a creatinine concentration of $1 \mathrm{mM}$ after the analysis. Fifty $\mu \mathrm{L}$ of the normalized extract from DUS were mixed in a glass tube with $20 \mu$ of the IS working solution, $130 \mu \mathrm{L}$ of reconstitution buffer, and vortexed.

\section{UHPLC-MS/MS}

Five $\mu \mathrm{L}$ of the finally urine or DUS samples respectively prepared as described in "Urine samples treatment" and "Dried urine spot (DUS) samples treatment" sections, were injected in the UHPLC system Agilent 1290 Infinity II (Agilent Technologies, CA, US) for the chromatographic analysis. The chromatography was performed with a Luna Omega SUGAR 100 column $\AA$, $150 \times 2.1 \mathrm{~mm}$ (Phenomenex, CA, US) at a flow rate of $0.5 \mathrm{~mL} / \mathrm{min}$. The mobile phase was a mixture of ammonium formate (Sigma-Aldrich Steinheim, Germany) $5 \mathrm{mM}+0.05 \%$ (v/v) FOA ( $>96 \%$ purity, reagent grade Sigma-Aldrich Steinheim, Germany) dissolved in water (A) and ammonium formate $5 \mathrm{mM}+0.05 \%(\mathrm{v} / \mathrm{v})$ FOA dissolved in $\mathrm{ACN}$ ( $\geq 99.9 \%$ purity for HPLC, gradient grade, Sigma-Aldrich Steinheim, Germany)/ water 90/10 (B). The gradient program is showed in Table 1 . The total run time was $27.50 \mathrm{~min}$ ( $15.50 \mathrm{~min}$ for detection and $12.00 \mathrm{~min}$ for column reconditioning) at a controlled column temperature of $40{ }^{\circ} \mathrm{C}$.

The UHPLC system was interfaced to a triple quadrupole 4500 SCIEX QTrap (AB Sciex, MA, US) equipped with a turbo ion spray source heated at $400{ }^{\circ} \mathrm{C}$. Nitrogen was used as curtain and collision gas. Common MS/MS parameters expressed in arbitrary units were the following: curtain gas (CUR), 20; ion source gas 1 (GS1), 20; ion source gas 2 (GS2), 20; collision-activated dissociation gas (CAD), 9; temperature (TEM), 400. In positive mode MS/MS parameters were: ion spray voltage (ISV), $5.500 \mathrm{~V}$; entrance potential (EP), $8 \mathrm{~V}$; cell exit potential (CXP), $10 \mathrm{~V}$; in negative mode MS/MS parameters were: ISV, $-4.500 \mathrm{~V}$; EP, $-8 \mathrm{~V}$; CXP, $-10 \mathrm{~V}$.

\section{Oligosaccharides detection}

MRM was used for spectra acquisition in positive and negative modes, switching the polarity within a single run. Data acquisition and chromatographic peak

\begin{tabular}{|c|c|c|c|}
\hline Time (min) & $A \%$ & B\% & $\begin{array}{l}\text { Flow rate } \\
(\mathrm{ml} / \mathrm{min})\end{array}$ \\
\hline 0 & 5 & 95 & 0.5 \\
\hline 5 & 60 & 40 & 0.5 \\
\hline 13.30 & 60 & 40 & 0.5 \\
\hline 13.50 & 30 & 70 & 0.5 \\
\hline 15.50 & 30 & 70 & 0.5 \\
\hline 17.50 & 5 & 95 & 0.5 \\
\hline 27.50 & 5 & 95 & 0.5 \\
\hline
\end{tabular}

A: ammonium formate $5 \mathrm{mM}+0.05 \%(\mathrm{v} / \mathrm{v})$ formic acid in water; $\mathrm{B}$ : ammonium formate $5 \mathrm{mM}+0.05 \%(\mathrm{v} / \mathrm{v})$ formic acid in acetonitrile/water $90 / 10$ 


\section{(See figure on next page.)}

Fig. 1 Chromatographic profiles of the examined storage disordes and of the internal standard (IS). The characteristic MRM transitions, in positive $(+)$ and negative $(-)$ modes, for each disorder are marked with different colours. Panel A: (1) extract ion chromatogram (XIC) of the seven positive MRM transitions characteristic for the IS maltoheptaose GIC7; (2) XIC of the seven negative MRM transitions characteristic for GIc7; (3) XIC of the three negative MRM transitions characteristic for sialidosis; (4) XIC of the eight positive MRM transitions characteristic for a-mannosidosis; (5) XIC of the nine positive MRM transitions characteristic for $\beta$-mannosidosis; (6) XIC of the eight negative MRM transitions characteristic for $\beta$-mannosidosis. Panel B: (7) XIC of the six positive MRM transitions characteristic for fucosidosis; (8) XIC of the two negative MRM transitions characteristic for Pompe disease, Vici syndrome, Yunis-Varon syndrome, and Danon disease; (9) XIC of the five positive MRM transitions characteristic for aspartylglucosaminuria; (10) XIC of the ten negative MRM transitions characteristic for aspartylglucosaminuria; (11) XIC of the five positive MRM transitions characteristic for GM1 gangliosidosis; (12) XIC of the seven positive MRM transitions characteristic for GM2 gangliosidosis

integration were conducted using the Analyst software (version 1.7 with HotFix $2,{ }^{\circledR} 2017$ AB SCIEX, Canada), using Glc7 as IS. For each sample, ratios of peak area/ Glc7 was calculated for single MRM transitions. For each MRM, the median (50th percentile) of at least 10 control samples was calculated and results were expressed as multiple of the medians (MoM).

The positive iQC was treated with the same procedure of samples and added to each batch series to control chromatography quality and the sensitivity of the tandem mass spectrometer.

\section{Results}

\section{Chromatography and mass spectra}

Figure 1 shows the extract ion chromatogram (XIC) of a selection of storage disorders (sialidosis, $\alpha$ - and $\beta$-mannosidosis, fucosidosis, aspartylglucosaminuria, GM1 and GM2 gangliosidosis, Pompe disease, Vici syndrome, Yunis-Varon syndrome and Danon disease) presenting a characteristic OS profile $[4,15]$.

\section{Assay validation}

For each MRM transition, linearity was estimated with calibration curves created using three samples at different urine concentrations and calculating the correlation coefficient $\left(R^{2}\right)$. The differently concentration samples were prepared by mixing and vortexing in a glass tube, as follow: $50 \mu \mathrm{L}$ of normalized ultra-filtered urine, $20 \mu \mathrm{L}$ of the IS working solution and $130 \mu \mathrm{L}$ of reconstitution buffer; $100 \mu \mathrm{L}$ of normalized ultra-filtered urine, $20 \mu \mathrm{l}$ of the IS working solution and $80 \mu \mathrm{L}$ of reconstitution buffer; $150 \mu \mathrm{L}$ of normalized ultra-filtered urine, $20 \mu \mathrm{L}$ of the IS working solution and $30 \mu \mathrm{L}$ of reconstitution buffer. In fresh urine, the $\mathrm{R}^{2}$ ranged from 0.8039 to 0.9999 in the positive mode and from 0.7270 to 0.9999 in the negative mode. In DUS, $\mathrm{R}^{2}$ ranged from 0.7701 to 0.9996 in the positive mode and from 0.8340 to 0.9995 in the negative mode.

For each MRM transition, the intra-day precision, expressed as $\mathrm{CV} \%$, was assessed by injecting and analyzing 10 times in the same run $5 \mu \mathrm{L}$ of the finally urine or DUS samples respectively prepared as described in
"Urine samples treatment" and "Dried urine spot (DUS) samples treatment" sections; the inter-day precision, expressed as $\mathrm{CV} \%$, was assessed by injecting and analyzing 10 times for three different days $5 \mu \mathrm{L}$ of the finally urine or DUS samples prepared respectively as described in "Urine samples treatment" and "Dried urine spot (DUS) samples treatment" sections.

The intra-day precision ranged from 6 to $24 \%$ in the positive and negative mode in fresh urine, from 5 to $25 \%$ in the positive mode and from 2 to $24 \%$ in the negative mode in DUS. The inter-day precision ranged from 9 to $25 \%$ in the positive mode and from 8 to $23 \%$ in the negative mode in fresh urine, from 7 to $26 \%$ in the positive mode and from 5 to $24 \%$ in the negative mode in DUS. Validation data of linearity, intra-day and inter-day precision in urine and DUS are reported in Additional file 1: A and $\mathrm{B}$.

\section{Detection of oligosaccharides in storage disorders}

The study allowed to confirm the diagnosis in all disorders presenting known OS profiles. Additional file 2: A and $B$ illustrate the MoM results obtained from all analyzed samples. Each sample was considered positive for a specific disorder when the MoMs of all qualifying and quantifying transitions were increased compared to controls at least 5 times for fresh urine and 2 times for DUS. Some disorders presented with a specific OS profile while in other diseases, the analysis revealed abnormal OS excretion but not-specific profiles. Overall, DUS samples showed lower background interferences when compared to urine samples.

\section{Disorders with a specific OS profile}

Figure 2 shows the scatter charts of the most characteristic transitions in disorders showing specific OS profiles [4].

Sialidosis Three negative MRM transitions were selected as the most characteristic for the sialyl-OS NeuAc-Hex3-HexNAc2 and one was used for quantitative analysis. We analyzed 1 urine and 1 DUS sample from one patient and both quantitative and qualitative 


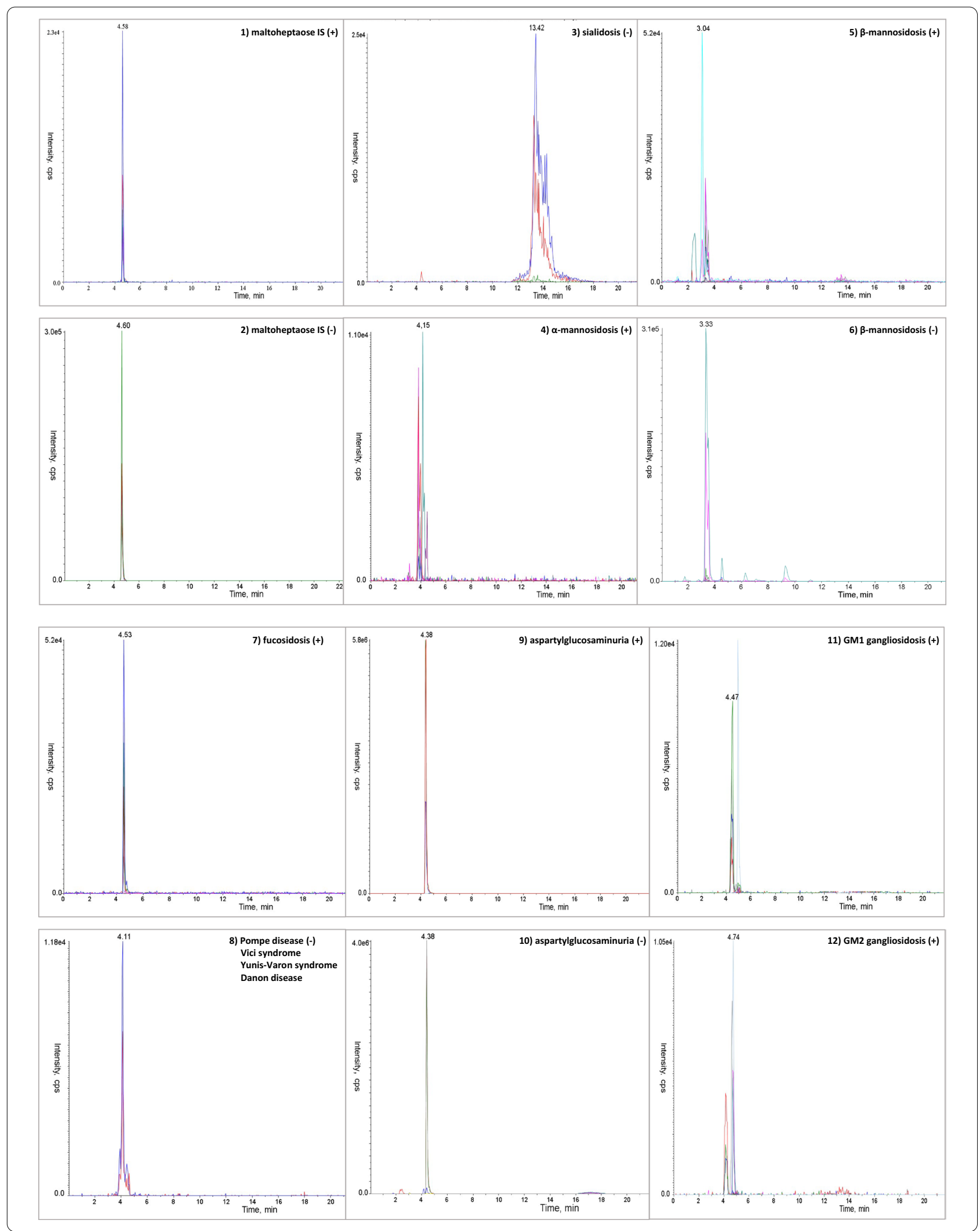


(See figure on next page.)

Fig. 2 Scatter charts of the most characteristic transitions in disorders showing specific OS profiles. The figure shows for each storage disorders the most characteristic MRM transitions in urine and DUS in comparison to controls: a sialidosis: transition 1200.4>1099.4 of the sialyl-OS NeuAc-Hex3HexNAc2; b a-mannosidosis: transition 568.2 > 347.2 of the mannosyl-OS NeuAc-Hex3HexNAc2; c fucosidosis: transition 504.2 > 289.2 of the fucosyl-OS Fuc-HexNAc-Asn; d $\beta$-mannosidosis: transition 406 > 244 of Hex-HexNAc and derivatives; e aspartylglucosaminuria: transition 520.2 > 305.2 of the GlcN-Asn + glycoasparaginyl-OS Hex-HexNAc-Asn; f GM1 gangliosidosis: transition 933.5 > 388.3 of the galactosyl-OS Hex3-HexNAc2; g GM2 gangliosidosis, Sandhoff type: transition 1136.3 > 933.4 of the N-acetylgalactosaminyl-OS Hex3-H; $\mathbf{h}$ GM2 gangliosidosis, Tay-Sachs type lacking the increase of the transition 1136.3 > 933.4 of the N-acetylgalactosaminyl-OS Hex3-HexNAc3 as seen in GM2 Sandhoff type

MRMs were highly increased compared to controls, with 102 fold elevation in urine and 668 fold in DUS.

$\alpha$-Mannosidosis Eight positive MRM transitions for the three mannosyl-OS, Hex2-HexNAc, Hex3-HexNAc and Hex4-HexNAc were selected as the most characteristic, and 4 were used for quantitative analysis. We analyzed 2 urine samples and 3 DUS obtained from 2 patients and all quantitative and qualitative MRMs were highly increased compared to controls, with a 110-118 fold elevation in urines and 29-245 fold in DUS for the most characteristic transition.

$\beta$-Mannosidosis Nine positive and eight negative MRM transitions for Hex-HexNAc and its derivatives were selected as the most characteristic, and 5 were used for quantitative analysis. We analyzed 1 urine sample and 1 DUS from a patient and all quantitative and qualitative MRMs were highly increased compared to controls, with 720 fold elevation in urine and 155 fold in DUS for the most characteristic transition.

Fucosidosis Six positive MRM transitions for the two fucosyl-OS, Fuc-HexNAc-Asn and Fuc-HexNAc2-Hex3, were selected as the most characteristic, and 3 were used for quantitative analysis. We studied 4 patients and analyzed 3 urine samples and 3 DUS and all quantitative and qualitative MRMs were highly increased compared to controls, with 220-470 fold elevation in urine and 152-373 fold in DUS for the most characteristic transition.

Aspartylglucosaminuria Five positive MRM transitions for aspartylglucosamine GlcN-Asn and the glycoasparaginyl-OS Hex-HexNAc-Asn and ten negative MRMs transitions for aspartylglucosamine GlcN-Asn and the glycoasparaginyl-OS NeuAc-Hex-HexNAc-Asn were selected as the most characteristic, and 3 positive and 3 negative MRMs were used for quantitative analysis. We studied 1 patient with aspartylglucosaminuria and analyzed 1 urine sample and 1 DUS and all quantitative and qualitative MRMs were highly increased compared to controls, with 71 fold elevation in urine and 80 fold in DUS for the most characteristic transition.
GM1 gangliosidosis Five positive MRM transitions for the two galactosyl-OS, Hex3-HexNAc2 and Hex5HexNAc3 were selected as the most characteristic, and 3 were used for quantitative analysis. We studied 7 patients and analyzed 14 urine samples and 4 DUS and all quantitative and qualitative MRMs were highly increased compared to controls, with 9-1160 fold elevation in urine and 69-883 fold in DUS for the most characteristic transition.

GM2 gangliosidosis (Sandhoff \& Tay-Sachs diseases) Seven positive MRM transitions for the three $\mathrm{N}$-acetylglucosaminyl-OS, Hex2-HexNAc2, Hex3HexNAc3 and Hex3-HexNAc4 were selected as the most characteristic of GM2 gangliosidosis $\mathrm{O}$ variant (Sandhoff disease) and 4 were used for quantitative analysis. We studied 4 Sandhoff patients and analyzed 6 urine and 1 DUS. In all samples, quantitative and qualitative MRMs were highly increased compared to controls, with 99-2102 fold elevation in urine and 79 fold in DUS for the most characteristic transition.

In the 3 patients with GM2 gangliosidosis B variant (Tay-Sachs disease) the OS analysis in urine and DUS did not display abnormalities.

Figure 3 shows the scatter charts of disorders presenting an increased excretion of Glc4 which included, besides Pompe disease, also the autophagy related disorders Vici and Yunis-Varon syndromes, and Danon disease.

Pompe disease (glycogen storage disease type II) Two negative MRM transitions of the tetrasaccharide Glc4 and of its isomer maltotetraose (M4) were selected as the most characteristic. We studied 3 untreated patients, two with infantile-onset and one with late-onset Pompe disease, and analyzed 4 urine and 2 DUS. Glc 4 was increased compared to controls, with a 10-72 fold elevation in urine and 11-13 fold in DUS, respectively. Lower levels were detected in the patient with milder phenotype.

Vici syndrome We studied 3 patients, one with the classic severe clinical picture and two sibs with a milder phenotype, and analyzed 4 urines and 3 DUS. The patient with the most severe variant showed an 21-28 fold increase of Glc4 in urine and an 18 fold increase in DUS, while the 
Semeraro et al. Orphaned J Rare Dis

(2021) $16: 24$

Page 7 of 11

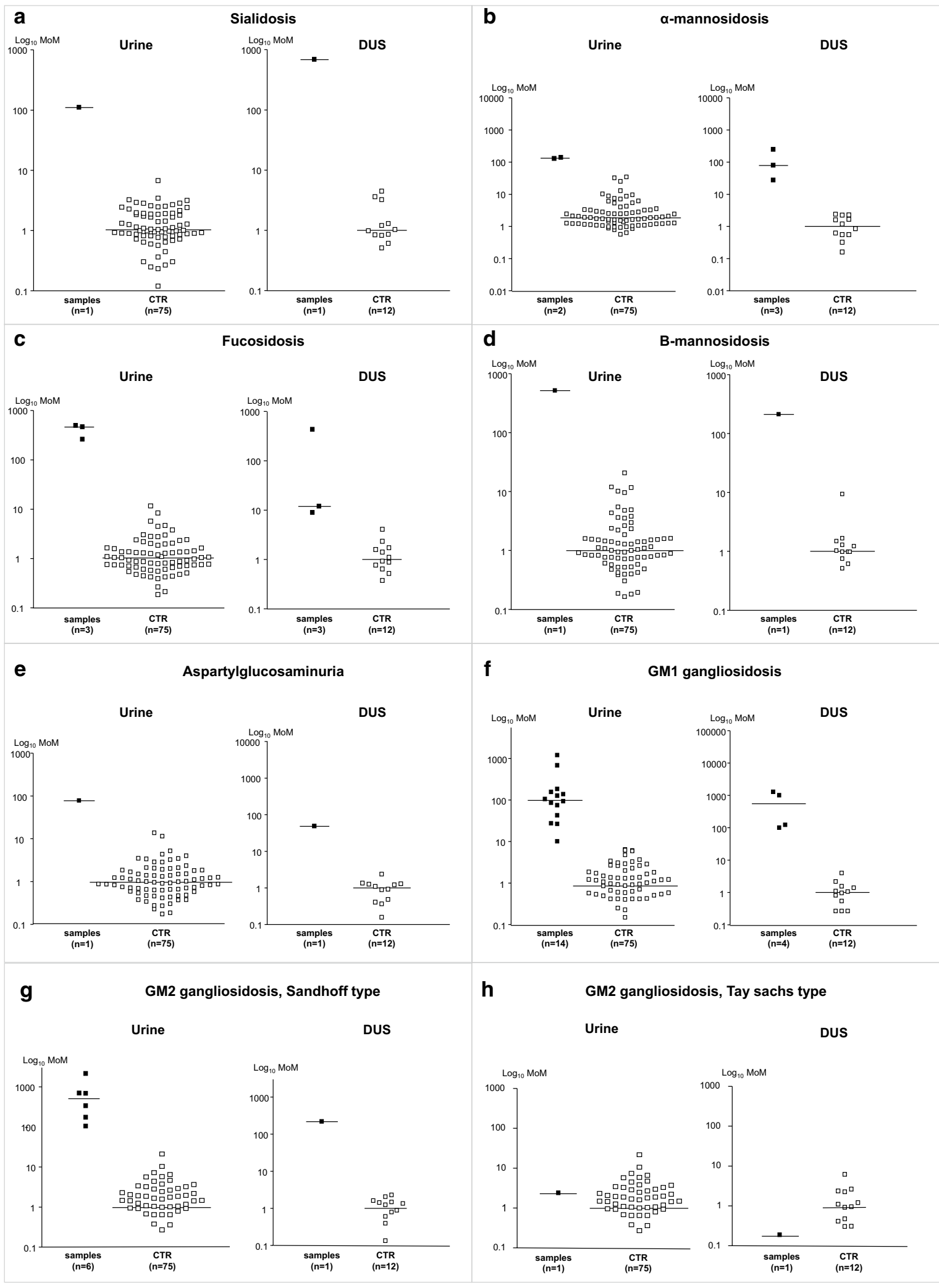



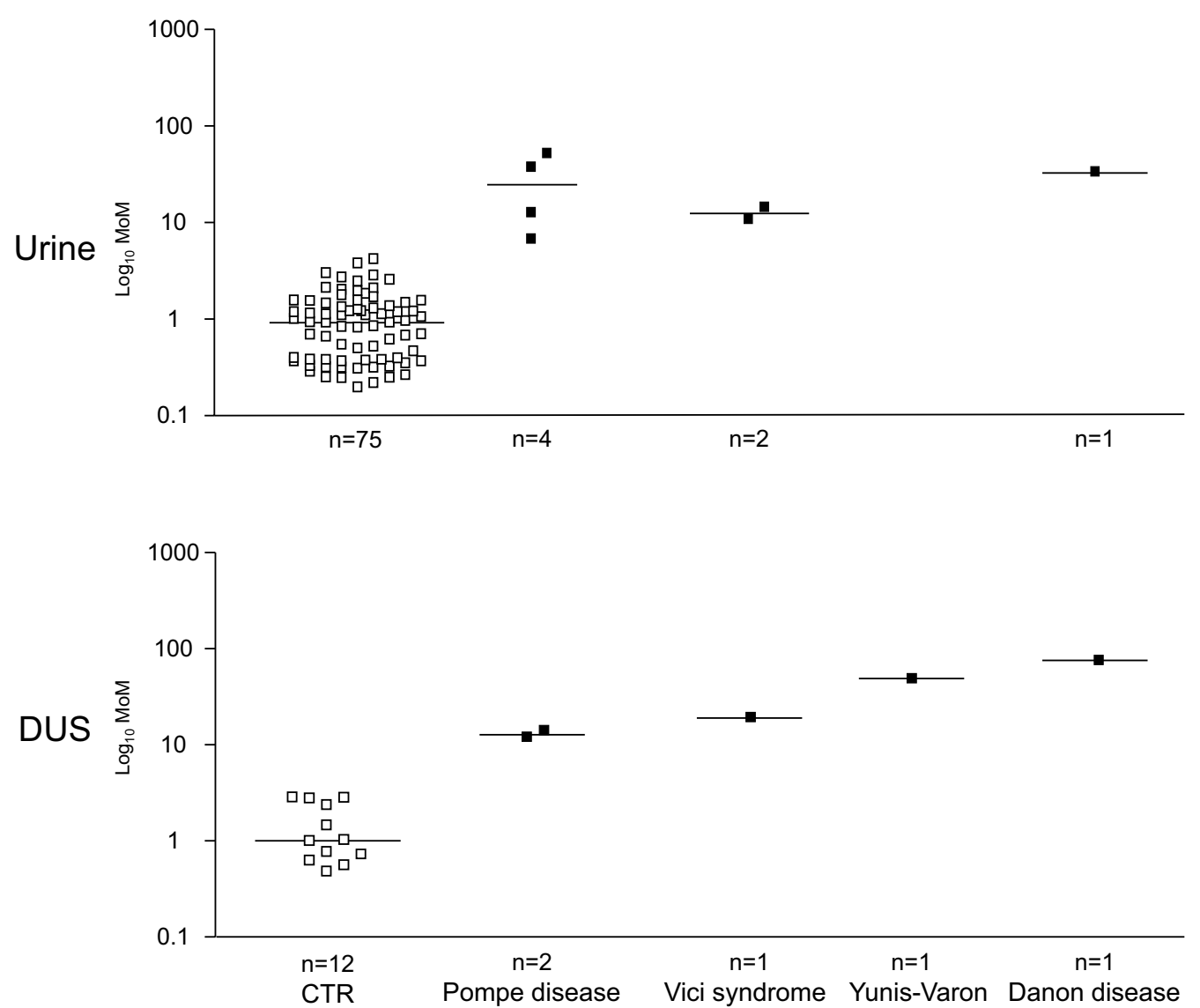

Fig. 3 Scatter charts of disorders presenting an increased excretion of Glc4 which included, besides Pompe disease, also the autophagy related disorders Vici and Yunis-Varon syndromes, and Danon disease. The figure shows the scatter charts in urine (top panel) and DUS (lower panel) of the transition $665>179$ of the tetrasaccharide 6-a-D-glucopyranosyl-maltotriose (Glc4) in Pompe disease, Vici syndrome, Yunis-Varon syndrome, and Danon disease in comparison to controls

two sibs with the less severe variant displayed only minor OS abnormalities both in urine and DUS and no elevation of Glc4.

Yunis-Varon syndrome The DUS sample from the patient with Yunis-Varon syndrome showed a 51 fold elevation of Glc4.

Danon disease (Glycogen storage disease type II B) We studied 1 patient with Danon disease and analyzed 1 urine and 1 DUS. Glc4 was increased compared to controls, with a 45 fold elevation in urine and 75 fold in DUS.

\section{Disorders with a not-specific OS profile}

Mucolipidosis type II, and type III We studied 2 patients with mucolipidosis type II and analyzed 3 urines and 1 DUS. In all urine samples OS analysis revealed mixed non specific profiles with $8 / 8$ positive transitions of $\alpha$-mannosidosis and $5 / 5$ positive transitions of GM1 gangliosidosis.
We studied 2 patients with mucolipidosis type III and analyzed 2 urines and 2 DUS. In urine $5 / 5$ positive MRM transitions of GM1 gangliosidosis were variably increased, however to a lesser extent than in GM1.

Mucopolysaccharidosis IVB DUS from 5 patients were available and all samples displayed increased levels of Glc4 however to a lesser extent than the positivity threshold.

\section{Discussion}

The diagnosis of storage disorders is often challenging due the presence of common clinical features and great variability in symptoms, and requires a complex approach which includes the analysis of target biomarkers in biological fluids, the measurement of enzymatic activities in leukocytes and/or fibroblasts and the confirmatory diagnosis by mutation analysis [20]. The availability of novel and specific therapies for a large number of diseases, including oligosaccharidoses, has increased the medical demand of reliable diagnostic techniques to 
Table 2 List of storage disorders screened with the UHPLC-MS/MS method

\begin{tabular}{|c|c|c|c|c|}
\hline Storage disorders & Enzyme/protein deficiency & Gene & MIM number & Characteristic oligosaccharides \\
\hline \multicolumn{5}{|l|}{ Oligosaccharidoses } \\
\hline Sialidosis & a-D-neuraminidase & NEU1 & 608272 & Sialyl-OS \\
\hline a-Mannosidosis & a-D-mannosidase & MAN2B1 & 609458 & Mannosyl-OS \\
\hline $\beta$-Mannosidosis & $\beta$-D-mannosidase & MANBA & 609489 & Hex-HexNAc and derivates \\
\hline Fucosidosis & a-ı-fucosidase & FUCA1 & 612280 & Fucosyl-OS \\
\hline Aspatylglucosaminuria & $N$-aspartyl- $\beta$-glucosaminidase & $A G A$ & 613228 & GlcN-Asn + glycoasparaginyl-OS \\
\hline \multicolumn{5}{|l|}{ Sphingolipidoses } \\
\hline GM1-gangliosidosis & $\beta$-D-galactosidase & GLB1 & 611458 & Galactosyl-OS \\
\hline GM2-gangliosidosis O variant (Sandhoff) & Hexosaminidase $\mathrm{A}$ and $\mathrm{B}$ & HEXB & 606873 & N-acetylgalacto saminyl-OS \\
\hline GM2-gangliosidosis B variant (Tay-Sachs) & Hexosaminidase A & $G M 2 A$ & 613109 & No abnormalities \\
\hline \multicolumn{5}{|l|}{ Glycogen storage disorders } \\
\hline Pompe disease (Glycogenosis type II) & Acid a-glucosidase & GAA & 606800 & Glc4 \\
\hline \multicolumn{5}{|l|}{ Disoders of autophagy } \\
\hline Vici syndrome & EPG5 & EPG5 & 615068 & Glc4 \\
\hline \multirow[t]{2}{*}{ Yunis-Varon syndrome } & FIG4 & FIG4 & 609390 & Glc4 \\
\hline & VAC 14 & VAC14 & 604632 & \\
\hline Danon disease (Glycogenosis type Ilb) & LAMP2 & LAMP2 & 309060 & Glc4 \\
\hline \multicolumn{5}{|l|}{ Mucolipidoses } \\
\hline Mucolipidosis type II \& III & N-acetylglucosamine-1-P-transferase & GNPTAB & 607840 & Non-specific abnormalities \\
\hline \multicolumn{5}{|l|}{ Mucopolisaccharidoses } \\
\hline Mucopolysaccharidosis IVB & $\beta$-D-galactosidase & GLB1 & 611458 & Non-specific abnormalities \\
\hline
\end{tabular}

offer precise and timely diagnosis. For this purpose, we developed a new UHPLC-MS/MS method for the screening of OS in urine and in DUS. The method was applied to screen known samples of a large panel of storage disorders and allowed to confirm the expected diagnoses (Table 2).

All examined oligosaccharidoses (i.e. sialidosis, $\alpha-/ \beta$ mannosidosis, fucosidosis, aspartylglucosaminuria) and both GM1 and GM2 (Sandhoff type only) gangliosidosis displayed specific OS profiles, as shown by the presence of single or multiple qualifying and quantifying MRM transitions [4]. In other diseases, such as mucolipidosis type II-III, and mucopolysaccharidosis type IVB the analysis revealed abnormal (non-specific) OS excretion, with mixed patterns combining MRM transitions of different disorders. Each OS was identified through its specific MRM transitions in positive and in negative modes [4]. Given the chemical similarity, we included in the OS analysis also the negative MRM transition of Glc4, the urinary biomarker of Pompe disease $[15,17]$. This implementation allowed to confirm the diagnosis of Pompe disease, with some variations in Glc4 excretion related to disease phenotypic severity.

Besides Pompe disease, Glc4 levels were markedly increased also in three disorders belonging to the autophagy machinery [21-23]. These include Vici syndrome and Yunis-Varon syndrome, two diseases characterized by a complex multisystem phenotype, and Danon disease, a disorder with clinical manifestations very similar to Pompe disease [24-28]. As seen in glycogen storage disorders, all these conditions share a cardiomuscular involvement with increased glycogen storage and variable vacuoles accumulation detectable on light microscopy at the level of skeletal and cardiac muscle,. In previous studies on Yunis-Varon syndrome, abnormalities of urinary OS by TLC analysis have been reported $[18,19]$. However, given the technical limitation of this method, a precise identification of these compounds was missed in the original reports. Our UHPLC-MS/MS method confirmed a relevant increase of OS excretion in Yunis-Varon syndrome and allowed to identify Glc4 as the disease-target compound. As a novel finding, upon evaluation of samples from patients with Vici syndrome and Danon disaese we detected a striking increase of Glc4 in urine. The structural and pathogenetic similarities with Pompe disease indicate that the increased urinary excretion of Glc4 in these three autophagy-related disorders reflects the abnormal muscle glycogen breakdown $[25,26]$, as also seen in other muscle disorders causing glycogen storage such as GSD type 3 and 6 [15, 29]. Glc4 could therefore serve as a biomarker to screen a wider range of storage disoders, including autophagyrelated diseases, presenting cardiomuscular sings with glycogen storage. 
From a technical perspective, most of recently reported methods using triple quadrupole for the analysis of OS require sample derivatization with 1-phenyl-3-methyl5-pyrazolone, making the preanalitycal phases of these methods time consuming $[3,8,9]$. Two MALDI-TOF/ TOF mass spectrometry methods, lacking internal standard, and suitable for disease screening have been reported $[11,12]$, with one not requiring sample derivatization [12].

The advantages of our screening method for OS analysis include a short preanalytical phase, not requiring sample derivatization, the use of UHPLC-MS/MS platform, a more widely available apparatus in diagnostic laboratories than MALDI-TOF/TOF mass spectrometry, and the possibility to perform OS analysis in urine and DUS, with comparable diagnostic results, thus making simpler and easier sample shipment and storage. The chromatography was performed with a HILIC phase column, which was chosen for its ability to improve retention and selective separation of sugar-related compounds. Unlike the more commonly used aminic columns, the HILIC column showed an enhanced lifetime because of the stationary phase composed with highly robust and thermally modified fully porous particles. The gradient was optimized to obtain the elution of all compounds in about $15 \mathrm{~min}$, with a residual $12 \mathrm{~min}$ for column reconditioning. The assay validation of the method in urine and DUS, showed that the correlation coefficient and the intra/inter-day precision of positive and negative MRM transitions were comparable in the two biological matrices. Moreover, in the control population, we noted that most of quantitative transitions showed significant differences for age below 6 month (12) versus eldest age (63) (see Additional file 3).

For each MRM, results obtained in patients and controls showed a clear separation of the two populations, both in urine and in DUS, without overlapping.

\section{Conclusions}

This new UHPLC/MS-MS method, not requiring sample derivatized and allowing the rapid and easy detection of OS in urine and DUS, expands the screening of storage disorders from oligosaccharidoses to other diseases, including Pompe disease and the novel category of inherited disorders of autophagy causing abnormal muscle glycogen breakdown.

\section{Supplementary Information}

The online version contains supplementary material available at https://doi. org/10.1186/s13023-020-01662-8.

Additional file 1: Table S1a. Validation data of positive MRM transitions; Table S1b. Validation data of negative MRM transitions.
Additional file 2: Table S2a. Results of urinary oligosaccharides analysis expressed as multiple of the median (MoM); Table S2b. Results of oligosaccharides analysis in DUS expressed as multiple of the median (MoM).

Additional file 3. Box plots showing, for the most characteristic transitions of storage disorders, significant differences in control groups for values $<6$ month and $>6$ month of age.

\section{Abbreviations}

OS: Oligosaccharides; DUS: Dried urine spots; TLC: Thin layer chromatography; MS: Mass spectrometry; MRM: Multiple reaction monitoring; HPAEC: High performance anion-exchange chromatography mass spectrometry; MALDITOF/TOF: Matrix-assisted laser desorption ionization time-of-flight; UHPLC-MS/ MS: Ultra-high performance liquid chromatography mass spectrometry; Glc4: Tetrasaccharide 6-a-D-glucopyranosyl-maltotriose; ERNDIM: European Research Network for Evaluation and Improvement of Screening, Diagnosis and Treatment of Inherited Disorders of Metabolism; iQC: Internal quality control; IS: Internal standard; GIc7: Maltoheptaose; ACN: Acetonitrile; FOA: Formic acid; CUR: Curtain gas; GS1: Ion source gas 1; GS2: Ion source gas 2; CAD: Collision-activated dissociation gas; TEM: Temperature; ISV: Ion spray voltage; EP: Entrance potential; CXP: Cell exit potential; MoM: Multiple of the median; $\mathrm{XIC}$ : Extract ion chromatogram; $\mathrm{R}^{2}$ : Correlection coefficient.

\section{Acknowledgements}

Thanks to Dr. Christine Vianey Saban acknowledging the use of data derived from ERNDIM EQA materials in this publication. The use of ERNDIM EQA materials does not imply that ERNDIM endorses the methods used or the scientific validity of the findings in this publication. ERNDIM (www.erndim.org) is an independent, not for profit foundation that provides EQA schemes in the field of inborn errors of metabolism with the aim of improving diagnosis, treatment and monitoring of inherited metabolic diseases. The authors would like to thank the "Associazione la Vita è un Dono ONLUS" and "Cbl-C ONLUS" for the research support to the Division of Metabolism; this work has been partly supported by the grant Ricerca Corrente from the Italian Ministry of Health. The Division of Metabolism is affiliated member of the European Reference Network for hereditary Metabolic Disorders (MetabERN).

\section{Authors' contributions}

MS designed and executed the experiments, performed data analysis and interpretation, and drafted the manuscript. Guarantor. ES designed and executed the experiments, performed data analysis and interpretation, and drafted the manuscript. FD contributed to select the samples and revised the manuscript. TC contributed to select the samples and revised the manuscript. IL contributed to select the samples, revised data and the manuscript. GC contributed to execute the experiments and revised the manuscript. GO contributed to selected the samples and revised the manuscript. CR contributed to analyse data and revised the manuscript. SB contributed to analyse data and revised the manuscript. CDV Contributed to experimental design, data analysis and data interpretation, drafted and revised the manuscript. All authors read and approved the final manuscript.

\section{Funding}

Ricerca corrente Italian Ministry of Health, Chiesi Farmaceutici, Sanofi Genzyme.

\section{Availability of data and materials}

Data supporting the findings of this study are available as electronic additional files.

\section{Ethical approval}

All procedures followed were in accordance with the ethical standards of the responsible committee on human experimentation (institutional and national) and with the Helsinki Declaration of 1975, as revised in 2000. The study has been approved by the Etics committee of the Bambino Gesù Childrens Hospital (n. 2119_OPBG_2020). All patients (or their guardians) approved of the possible use of their left-over samples for method validation purposes, in agreement with institutional and national legislation. This article does not contain any studies with animal subjects performed by the any of the authors. 


\section{Consent for publication \\ Not applicable.}

\section{Competing interests}

The authors declare that they have no competing interests.

\begin{abstract}
Author details
${ }^{1}$ Division of Metabolism and Metabolic Diseases Research Unit, Bambino Gesù Children's Hospital, IRCCS, Viale San Paolo 15, 00146 Rome, Italy. ${ }^{2}$ Metabolism Unit, Department of Pediatrics, Faculty of Medicine, Hacettepe University, Ankara, Turkey. ${ }^{3}$ Department of Medical Biochemistry and Hacettepe University Hospitals Clinical Pathology Laboratory, Faculty of Medicine, Hacettepe University, Ankara, Turkey.
\end{abstract}

Received: 25 June 2020 Accepted: 22 December 2020

Published online: 09 January 2021

\section{References}

1. Ferreira CR, van Karnebeek CDM, Vockley J, Blau N. A proposed nosology of inborn errors of metabolism. Genet Med. 2019;21:102-6.

2. Ferreira CR, Gahl WA. Lysosomal storage diseases. Transl Sci Rare Dis. 2017;2:1-71.

3. Ramsay SL, Meikle PJ, Hopwood JJ, Clements PR. Profiling oligosaccharidurias by electrospray tandem mass spectrometry: quantifying reducing oligosaccharides. Anal Biochem. 2005;345:30-46.

4. Piraud M, Pettazzoni M, Menegaut L, et al. Development of a new tandem mass spectrometry method for urine and amniotic fluid screening of oligosaccharidoses. Rapid Commun Mass Spectrom. 2017;31:951-63.

5. Sewell AC. Urinary oligosaccharide excretion in disorders of glycolipid, glycoprotein and glycogen metabolism. A review of screening for differential diagnosis. Eur J Pediatr. 1980;134:183-94.

6. Humbel R, Collart M. Oligosaccharides in urine of patients with glycoprotein storage diseases.Rapid detection by thin-layer chromatography. Clin Chim Acta. 1975;60:143.

7. Kurczynski TW, Kendzierski KS, Sewell AC, Kuczynski TW. Urinary oligosaccharides in pregnant or lactating women: pitfall in screening. Clin Chem. 1993;39:2346-7.

8. Ramsay SL, Maire I, Bindloss C, et al. Determination of oligosaccharides and glycolipids in amniotic fluid by electrospray ionisation tandem mass spectrometry: in utero indicators of lysosomal storage diseases. Mol Genet Metab. 2004;83:231-8.

9. Sowell J, Wood T. Towards a selected reaction monitoring mass spectrometry fingerprint approach for the screening of oligosaccharidoses. Anal Chim Acta. 2011;686:102-6.

10. Bruggink C, Poorthuis BJ, Deelder AM, Wuhrer M. Analysis of urinary oligosaccharides in lysosomal storage disorders by capillary high-performance anion-exchange chromatography-mass spectrometry. Anal Bioanal Chem. 2012;403:1671-83.

11. Xia B, Asif G, Arthur L, et al. Oligosaccharide analysis in urine by maldi-tof mass spectrometry for the diagnosis of lysosomal storage diseases. Clin Chem. 2013;59:1357-68.

12. Bonesso L, Piraud M, Caruba C, Van Obberghen E, Mengual R, Hinault C. Fast urinary screening of oligosaccharidoses by MALDI-TOF/TOF mass spectrometry. Orphanet J Rare Dis. 2014;9:19.
13. Huang R, Cathey S, Pollard L, Wood T. UPLC-MS/MS analysis of urinary free oligosaccharides for lysosomal storage diseases: diagnosis and potential treatment monitoring. Clin Chem. 2018;64:1772-9.

14. Young SP, Zhang H, Corzo D, et al. Long-term monitoring of patients with infantile-onset Pompe disease on enzyme replacement therapy using a urinary glucose tetrasaccharide biomarker. Genet Med. 2009;1 1:536-41.

15. Sluiter W, van den Bosch J, Goudriaan DA, et al. Rapid ultraperformance liquid chromatography-tandem mass spectrometry assay for a characteristic glycogen-derived tetrasaccharide in Pompe disease and other glycogen storage diseases. Clin Chem. 2012;58:1139-47.

16. Young SP, Piraud M, Goldstein JL, et al. Assessing disease severity in Pompe disease: the roles of a urinary glucose tetrasaccharide biomarker and imaging techniques. Am J Med Genet C Semin Med Genet. 2012;160:50-8.

17. Canbay E, Vural M, Kalkan Uçar S, et al. The decision-making levels of urine tetrasaccharide for the diagnosis of Pompe disease in the Turkish population. J Pediatr Endocrinol Metab. 2020;33:391-5.

18. Dworzak F, Mora M, Borroni C, et al. Generalized lysosomal storage in Yunis-Varon syndrome. Neuromuscul Disord. 1995;5:423-8.

19. Walch E, Schmidt M, Brenner RE, et al. Yunis-Varon syndrome: evidence for a lysosomal storage disease. Am J Med Genet. 2000;95:157-60.

20. Mashima R, Okuyama T, Ohira M. Biomarkers for lysosomal storage disorders with an emphasis on mass spectrometry. Int J Mol Sci. 2020;21:2704.

21. García-Cazorla À, Saudubray JM. Cellular neurometabolism: a tentative to connect cell biology and metabolism in neurology. J Inherit Metab Dis. 2018:41:1043-54.

22. Levine B, Kroemer G. Biological functions of autophagy genes: a disease perspective. Cell. 2019;176:11-42.

23. Teinert J, Behne R, Wimmer M, Ebrahimi-Fakhari D. Novel insights into the clinical and molecular spectrum of congenital disorders of autophagy. J Inherit Metab Dis. 2020;43:51-62.

24. Cullup T, Kho AL, Dionisi-Vici C, et al. Recessive mutations in EPG5 cause Vici syndrome, a multisystem disorder with defective autophagy. Nat Genet. 2013;45:83-7.

25. Byrne S, Dionisi-Vici C, Smith L, Gautel M, Jungbluth H. Vici syndrome: a review. Orphanet J Rare Dis. 2016;11:21.

26. Campeau PM, Lenk GM, Lu JT, et al. Yunis-Varon syndrome is caused by mutations in FIG4, encoding a phosphoinositide phosphatase. Am J Hum Genet. 2013;92:781-91.

27. Lines $M A$, Ito $Y$, Kernohan $K D$, et al. Yunis-Varon syndrome caused by biallelic VAC14 mutations. Eur J Hum Genet. 2017;25:1049-54.

28. Veinot JP, Nair V. Lysosomal storage disorders affecting the heart: a review. Cardiovasc Pathol. 2020;48:107217.

29. Farah BL, Yen PM, Koeberl DD. Links between autophagy and disorders of glycogen metabolism - perspectives on pathogenesis and possible treatments. Mol Genet Metab. 2020;129:3-12.

\section{Publisher's Note}

Springer Nature remains neutral with regard to jurisdictional claims in published maps and institutional affiliations.

Ready to submit your research? Choose BMC and benefit from:

- fast, convenient online submission

- thorough peer review by experienced researchers in your field

- rapid publication on acceptance

- support for research data, including large and complex data types

- gold Open Access which fosters wider collaboration and increased citations

- maximum visibility for your research: over 100M website views per year

At BMC, research is always in progress.

Learn more biomedcentral.com/submissions 